\title{
Combined Therapy with Dendritic Cell Loaded- Exosomes Supplemented with PD-1 Inhibition Have Superior Antitumor Effect in Hepatocellular Carcinoma
}

\section{Shengbin Shi}

Shandong Cancer Hospital: Shandong Cancer Hospital and Institute

\section{Cuijuan Wang}

Shandong First Medical University

Chuangnian Zhang

Chinese Academy of Medical Sciences \& Peking Union Medical College Institute of Biomedical

Engineering

\section{Wenyu Zhang}

Shandong Cancer Hospital: Shandong Cancer Hospital and Institute

\section{Yibo Qin}

Chinese Academy of Medical Sciences \& Peking Union Medical College Institute of Biomedical

Engineering

\section{Zuoxing Niu ( $\nabla$ nzxsdth@163.com )}

Shandong Tumor Hospital and Institute

\section{Research}

Keywords: PD-1 antibody, Exosomes, Dendritic cells, Hepatocellular carcinoma

Posted Date: July 7th, 2021

DOl: https://doi.org/10.21203/rs.3.rs-638732/v1

License: (c) (i) This work is licensed under a Creative Commons Attribution 4.0 International License. Read Full License 


\section{Abstract}

Background Hepatocellular carcinoma (HCC) is a common cause of cancer-related deaths and has low sensitivity to conventional therapies. Dendritic cell (DC) loaded tumor-exosomes (TEX) have shown antitumor effects in a murine HCC model. However, the combined antitumor efftcts of DC-TEX and programmed death protein 1 antibody (PD-1 Ab) at different time application has not been investigated.

Methods In this study, ectopic, orthotopic, and diethylnitrosamine (DENA)-induced HCC models were established and treated with DC-TEX alone or in combination with PD-1 Ab at different time points. Meanwhile, we established an orthotopic HCC model in BALB/C nude mice and restore t cells.

Results The results showed that along with the increased number of CD8+ T cells, the PD- $1+C D 8+T$-cell population was also significantly increased after DC-TEX injection. The number of CD8+ T cells peaked $72 \mathrm{~h}$ after DC-TEX injection, and the PD-1+CD8+ T cells also showed a similar result. Subsequently, the PD-1 Ab was applied in combination with DC-TEX at a series of time points $(0,24,72,96,120$ or $168 \mathrm{~h})$. Surprisingly, the combined treatment showed strong antitumor effects and this effect was most prominent when PD-1 Ab was administerd at $72 \mathrm{~h}$. In vitro, PD-1 Ab also significantly reversed the proliferative ability of PD-1+CD $8+T$ cells at $72 \mathrm{~h}$. The combined antitumor effects of PD-1 Ab and DCTEX were mainly through their stimulation on $C D 8+T$ cells proliferation as well as repression for $T$ cell exhaustion.

Conclusion In conclusion, the combination of DC-TEX and PD-1 Ab significantly suppresses tumor growth in a murine HCC model and that the antitumor effect is affected by the timing of PD-1 Ab treatment.

\section{Background}

Hepatocellular carcinoma (HCC) is one of the most malignant cancers, with a high rate of mortality and increasing incidence over the last two decades $[1,2]$. Unfortunately, many patients with early-stage HCC are asymptomatic and can only be diagnosed at intermediate and advanced stages. Nevertheless, patients with advanced HCC barely respond to regular chemotherapy or radiotherapy. Sorafenib is a multikinase inhibitor and is currently the only systemic therapy for advanced HCC approved by the US Food and Drug Administration. However, most patients rapidly become sorafenib-resistant, with a survival time of less than 3 months $[3,4]$. Therefore, effective treatment for advanced HCC is critically needed.

Recently, immunotherapy has been presented as a promising emerging treatment for HCC in several studies [5]. One study has demonstrated that defective T-cell responses and functional defects of antigen presentation significantly decreased the immune function of HCC patients [6]. At the same time, the number of T-infiltrating lymphocytes in the tumor tissue may predict the prognosis of HCC patients [7]. Tumor-associated antigens are especially crucial in immunotherapy. Dendritic cells (DCs) are a type of antigen-presenting cells and possess the unique ability to process and present antigens that can elicit tissue-specific T-cell responses and change the tumor microenvironment $[8,9]$. 
One important pathway to activate antigen-presenting DCs by tumors is via tumor-released exosomes (TEX).Exosomes are small-membrane vesicles (30-100 nm) secreted by most cell types, including tumor cells [10]. TEXs express tumor-specific antigens that can be processed by DCs and then activate DCs. Exosome-pulsed DCs can induce antigen-specific CD8 + T cells with antitumor effects [11]. Interestingly, when tumor-derived exosomes were used as vaccines, the immunogenicity was even higher than that of tumor cell lysates in a murine HCC model [12].

With chronic antigen exposure, tumor antigen-specific CD $8+T$ cells become dysfunctional and exhausted, and lose the ability to proliferate and produce cytokines [13]. The dysfunctional tumor antigen-specific CD8 + T cells upregulate immunoinhibitory receptors, including programmed cell death protein 1 (PD-1) [14]. PD-1, a 55-kD type I transmembrane protein of the immunoglobulin super family, is an immunoinhibitory receptor whose expression is inducible in activated T cells. In a tumor microenvironment, tumor antigen may induce PD-1 expression on CD8 + T cells, and these PD- $1+C D 8+T$ cells exhibit an impaired ability to produce effector cytokines upon binding with programmed death ligand 1 (PD-L1) [15-16]. In patients with melanoma, the interactions of PD-1/PD-L1 not only impaired the ability of spontaneously induced specific CD8 $+T$ cells but also vaccine-induced specific CD $8+T$ cells[17]. Abundant experimental and clinical evidence have provedthat blocking the PD-1 axis reversed the dysfunction and exhaustion of activated $T$ cells and presented a significant benefit for the tumor microenvironment by enabling the exhausted $T$ cells to proliferate and produce effector cytokines $[18,19]$.

Based on the characteristics of DC-TEX and PD-1, we postulated that combination treatment with DC-TEX and a PD-1 antibody would improve antitumor responses. We examined the feasibility of these two treatment methods in $\mathrm{HCC}$ models and how to use the method to improve antitumor efficacy. we found that the combined treatment performed much better than DC-TEX treatment alone. We also found that the timing of PD-1 antibody treatment significantly impacted the antitumor effects of the combined therapy.

\section{Materials And Methods}

Cell lines

The murine HCC cell line Hepa1-6 was purchased from the Cell Bank of the Chinese Academy of Sciences (shanghai china) and was cultured in Dulbecco's modified Eagle's medium (DMEM; HyClone) supplemented with $10 \%$ fetal bovine serum (FBS; Gibco) and $1 \%$ penicillin-streptomycin in a $37^{\circ} \mathrm{C}$ incubator with $5 \% \mathrm{CO}_{2}$. The murine cell line DC2.4 (DCs) was kindly provided by Dr. De Yang (Center for Cancer Research, National Institutes of Health, Bethesda, MD) and was cultured as described previously [12].

Mice and HCC model

C57BL/ 6 mice, BALB/C mice, and BALB/C nude mice (4 to 6 weeks old) were purchased from Beijing Vital River Laboratory Animal Technology Co. Ltd. and were kept under pathogen-free conditions in the Tianjin Animal Unit (SYXK 2011-0008). Animal studies were approved by the Experimental Animal 
Management Committee of the Chinese Academy of Medical Sciences and Peking Union Medical College Institute of Biomedical Engineering. The orthotopic HCC model was established using tissue implantation as previously described [12]. Briefly, Hepa1-6 cells $\left(4 \times 10^{6} / 50 \mu \mathrm{L}\right)$ were injected subcutaneously in the left axilla of mice. When the tumor grew to $1 \mathrm{~cm}$ in diameter, it was excised and cut into tumor blocks of approximately $1 \mathrm{~mm}^{3}$, and necrotic tissue was removed. Two tumor blocks were implanted into the left lobe of the liver of a C57BL/ 6 mouse or a BALB/C nude mouse under celiac anesthesia with $4 \%$ chloral hydrate. To establish autochthonous HCC mouse models, each of the 15-day-old C57BL/ 6 mice received a single dose of $50 \mu \mathrm{g} / \mathrm{g}$ DENA (Sigma) by intraperitoneal injection and was then used in this study after 8 months. In BALB/C nude mice, the properties of T cells were restored by injecting $T$ cells derived from the thymus gland of BALB/C mice (4 weeks old).

\section{Treatment}

Fourteen days after tissue implantation, mice with an established orthotopic HCC tumor confirmed by magnetic resonance imaging (MRI, 3.0 Tesia, Signa Excite HDx; GE healthcare, Milwaukee, WI, USA) were randomized by tumor size to assure similar baseline tumor sizes across groups. For autochthonous HCC mouse models, the treatment was initiated 8 months after the injection of DENA. The tumor volume was calculated using the following formula: tumor volume $\left(\mathrm{mm}^{3}\right)=a \times b^{2} \times 0.5$, where a represents the longest diameter and $b$ represents the shortest diameter. Mice were treated with DC-TEX $\left(4 \times 10^{6}\right.$, intravenous injection, DCs pulsed with exosomes $(40 \mu \mathrm{g} / \mathrm{mL})$ for $48 \mathrm{~h})$ and/or PD-1 Ab $(200 \mu \mathrm{g}$, intraperitoneal injection, clone J43; BioXCell, West Lebanon, NH, USA).

Generation and purification of exosomes and loading of DCs with tumor exosomes

Exosomes were isolated from Hepa1-6 cell culture supernatant by ultracentrifugation as described previously [18]. Briefly, centrifugation at $2000 \times \mathrm{g}$ for $20 \mathrm{~min}$ and then $10,000 \times \mathrm{g}$ for $30 \mathrm{~min}$ was performed to discard dead cells and cell debris and was followed by ultracentrifugation at $100,000 \times \mathrm{g}$ for 70 min (Hitachi, Tokyo, Japan). The exosome pellets were washed with PBS and then centrifuged at $100,000 \times \mathrm{g}$ for $70 \mathrm{~min}$ before re-suspension in PBS. The protein concentration was determined using a BCA protein assay kit (Pierce, USA) and was adjusted to $1 \mu \mathrm{g} / \mu \mathrm{L}$ before storage at $-80^{\circ} \mathrm{C}$ until use. To prepare DC-TEX, DCs were pulsed with exosomes $(40 \mu \mathrm{g} / \mathrm{mL})$ for $48 \mathrm{~h}$ in DMEM, which allows the DCs to capture and present the tumor-associated antigens. The concentration of exosomes was previously confirmed [12], and DCs that were pulsed with exosomes $(40 \mu \mathrm{g} / \mathrm{mL})$ showed a superior antitumor effect in orthotopic murine HCC models.

Flow cytometry analysis

Flow cytometry analysis was performed to detect the number of $\mathrm{T}$ lymphocytes in tumor tissue. A singlecell suspension of tumor tissue was generated, as described previously, applying a digestion with collagenase type IV ( $0.05 \mathrm{mg} / \mathrm{mL}$; Gibco, Invitrogen) and $40 \%$ Percoll (Solarbio, Beijing, China) to enrich for the tumor-infiltrating lymphocytes [12]. The collected cells were stained with fluorescent-labeled monoclonal antibodies: FITC-conjugated CD3e (clone 145-2C11), APC-conjugated CD8a (clone 53 - 6.7), 
PE-conjugated PD-L1 (clone MIH6) and PE-conjugated PD-1 (clone J43). All antibodies were purchased from eBioscience (CA, USA) and were detected with a flow cytometer with a four color BD Accuri C6 (BD Biosciences, CA). Analysis was performed using BD Accuri C6 software (BD biosciences, CA).

Immunohistochemistry

Tumor tissues were routinely embedded in paraffin and then cut into 4- $\mu$ m-thick sections for immunohistochemical staining of PD-L1 and CD8. The tumor sections were first deparaffinized in xylene and then rehydrated through a graded alcohol series. They were then subjected to antigen retrieval performed at $100^{\circ} \mathrm{C}$ in citrate buffer for $30 \mathrm{~min}$ and treated with $3 \% \mathrm{H}_{2} \mathrm{O}_{2}$ for 10 min to inhibit endogenous peroxidase activity. The sections were blocked with $10 \%$ goat serum for $15 \mathrm{~min}$ and then stained with primary anti-mouse PD-L1 (ab205921, 1:300; Abcam) or CD8 (ab22378,1:1000; Abcam) polyclonal antibody overnight at $4^{\circ} \mathrm{C}$. The next day, the sections were treated with secondary antibody (KPL, 074-1506, antibody to rabbit lgG) for $30 \mathrm{~min}$ at $37^{\circ} \mathrm{C}$ and then stained with diaminobenzidine followed by counterstaining with hematoxylin.

Cytokine detection using enzyme-linked immunosorbent assay (ELISA)

The serum levels of cytokines, including interleukin-2 (IL-2), interleukin-10 (IL-10), tumor necrosis factor-a (TNF-a), interferon- $\gamma$ (IFN- $\gamma$ ), transforming growth factor- $\beta$ (TGF- $\beta$ ), and a-fetoprotein (AFP) were measured using ELISA assay kits (eBioscience, CA, USA) following the manufacturer's instructions.

Statistical analysis.

For statistical analysis, all data are presented as the mean \pm standard deviation. The statistical significance of differences between groups were assessed using ANOVA. We chose $P<0.05$ in a twotailed test to indicate statistical significance. Overall survival was evaluated using the Kaplan-Meier method. Analyses were performed with SPSS 16.0 software (SPSS Inc., Chicago, IL).

\section{Results}

DC-TEX could increase the number of CD8 $+T$ cells and upregulate the levels of cytokines in orthotopic HCC mice

To verify the if DC-TEX activate $T$ cell immune response, we first examined the number of CD $8+T$ cells and effector cytokine production level after DC-TEX treatment in a orthotopic mouse model.We observed variations in the levels of CD8 $+T$ cells and cytokines after injection of DC-TEX (exosome characteristics are presented in Supplementary Figure S1A, B) in orthotopic HCC mice. The number of CD $8+T$ cells in tumor tissue was detected by flow cytometry at $0,24,72,120$ and $168 \mathrm{~h}$ after injection with DC-TEX. The results demonstrated that the number of CD8 + T cells in tumor tissue increased initially and peaked at $72 \mathrm{~h}$, while at $120 \mathrm{~h}$, the number started to decrease(Fig. 1A,B). Meanwhile, we evaluated the level of cytokines, including IFN- $\gamma$, IL-2, TNF- $a$, IL-10, and TGF- $\beta$ using ELISA. The process of T-cell activation with 
DC-TEX increased the production of several cytokines, including IFN-y, IL-2 and TNF- $a$. Interestingly, the levels of IFN-y, IL-2 and TNF-a also peaked at $72 \mathrm{~h}$ time point (Fig. 1C-E). DC-TEX has been reported to change the tumor environment by decreasing the level of IL-10 and TGF- $\beta$. In this study, we also found that the levels of IL-10 and TGF- $\beta$ were decreased after DC-TEX treatment (Fig. 1F, G).

PD-1 + CD8 + T cells were significantly elevated $72 \mathrm{~h}$ after injection of DC-TEX

To know when CD8 + T might reach exhaustion, we analyzed PD-1 + CD 8 + T cells in tumor tissues derived from HCC model mice by flow cytometry at different time points after DC-TEX treatment. The PD-1 + CD8 $+T$ cell number peaked at $72 \mathrm{~h}$ after therapy with DC-TEX in the tumor issue (Fig. 2A, B). The number of PD $-1+C D 8+T$ cells at $72 \mathrm{~h}$ was higher than the number at 0 and $24 \mathrm{~h}(P<0.05)$, whereas the number of PD- $1+$ CD $8+T$ cells was not increased significantly at $120 \mathrm{~h}$ and $168 \mathrm{~h}$ when compared with the $72 \mathrm{~h}$ time point and shown a downward trend $(P>0.05)$. Additionally, we applied Mouse lgG1 $\mathrm{K}$ Isotype Control PE as PD-1 antibody, but there was no significant difference at different time points (Supplementary Figure S2 A, B). We co-cultured T cells from the spleen of C57 mice with DC-TEX and then observed the expression of PD-1 at different time points from 0-168 h. We found that the expression of PD-1 increased gradually during the activation of T cells (Fig. 2C, Supplementary Figure S3) from 0 to $72 \mathrm{~h}(P<$ 0.05). The mRNA of PD-1 (PDCD1) showed highest expression at $72 \mathrm{~h}$ (Fig. 2D) by qRT-PCR. Consistently, PD-1 protein also demonstrated highest expression of PD-1 at $72 \mathrm{~h}$ via western blot (Fig. 2E).

To confirm the results independently, we developed an orthotopic HCC model in immune-deficient BALB/C nude mice. We first restored BALB/C nude mice $T$ cell population by injecting $T$ cells derived from the thymus of BALB/C mice. We then applied DC-TEX (DCs derived from the bone marrow of BALB/C mice) to these mice and observed T cell proliferation and IFN-y production post treatment. We found that both observations recapitulated previous results: both the PD-1 + CD8 + T-cell numbers in the tumor tissues as well as the cytokine IFN- $y$ level peaked at $72 \mathrm{~h}$ (Supplementary Figure S4).

Treatment with PD-1 Ab at different time points after DC-TEX injection has different antitumor efficacies

So far, we have shown DC-TEX treatment significantly stimulated CD8 $+T$ cell proliferation as well as its cytokine production. We have also shown evidence of T cell exhaustion via its expression of PD-1.

Therefore, we set out to test if a combined treatment with DC-TEX and PD-1 inhibition would give better antitumor effect. we assessed the efficacy of PD-1 Ab administration starting at 0, 24, 72, 120 and $168 \mathrm{~h}$ after injection of DC-TEX. On the 14th day after implantation of tumor tissue into C57 mice, we performed MRI to confirm the sizes of tumors in established orthotopic HCC model mice (Supplementary Figure S5 $A, B)$ prior to DC-TEX injection. The treatment schedule is presented in Supplementary Figure S6. DC-TEX $\left(4 \times 10^{6}\right)$ was injected intravenously and $200 \mu \mathrm{g}$ PD-1 Ab was injected intraperitoneally three times at weekly intervals. On day 40, we evaluated the tumor weights and the $72 \mathrm{~h}$ group presented a superior result (Fig. 3A, B). Additionally, the number of CD8 + T cells (Fig. 3C) and the level of IFN-y, IL2, and TNF$\mathrm{a}$ (Supplementary Figure S7 A-C) were elevated significantly in the $72 \mathrm{~h}$ group. We detected the level of alpha-fetoprotein (AFP) using an ELISA kit and found that the level in the $72 \mathrm{~h}$ group was significantly 
lower than in other groups (Fig. 3D). Furthermore, we observed the survival times of all groups, and the 72 $\mathrm{h}$ group showed significantly prolonged survival (Fig. 3E). These results demonstrated that the timing of PD-1 Ab administration after DC-TEX injection significantly influenced the antitumor efficacy of the combined therapy.

\section{PD-1 antibody reversed the proliferative ability of PD-1 + CD8 + T cells}

To verify if PD-1 inhibition reversed the proliferative ability of PD-1 + CD $8+T$ cells, in vitro, we separated $\mathrm{CD} 8+\mathrm{T}$ cells from the spleens of C57 mice by magnetic activated cell sorting and then cocultured them with DC-TEX(Supplementary Figure S8). CD8 + T cells derived from spleen were labeled with 5,6carboxyfluoresceindiacetate,succinimidyl ester(CFSE) and cocultured with DC-TEX. Subsequently, PD-1 Ab was added to the coculture at different time points ( 0 to $168 \mathrm{~h}$ ). The proliferation of CD $8+T$ cells was significantly increased at $72 \mathrm{~h}$ (Fig. 4A,B). Additionally, the effective cytolysis against Hepa1- 6 cells showed a significant difference for different effector to target ratios, with treatment for $72 \mathrm{~h}$ demonstrating a superior antitumor effect (Fig. 4C). Subsequently, we selected the $0 \mathrm{~h}$ and $72 \mathrm{~h}$ time points to analyze granzyme $B+C D 8+T$ cells and perforin $+C D 8+T$ cells by flow cytometry, with significantly higher levels observed at $72 \mathrm{~h}$ than $0 \mathrm{~h}$ (Fig. 4D, E). Additionally, the number of IFN- $\mathrm{C}+\mathrm{CD} 8+$ $\mathrm{T}$ cells and TNF- $\mathrm{a}+\mathrm{CD} 8+\mathrm{T}$ cells was higher at $72 \mathrm{~h}$ than $0 \mathrm{~h}$ (Supplementary Figure S9). This confirmed the varying antitumor effects at different time points using PD-1Ab.

The Antitumor effect of PD-1 Ab and DC-TEX is mainly dependent on CD $8+T$ cell

The antitumor effect of DC-TEX is mediated by CD8 + T cells. This is demonstrated by the fact that DCTEX does not have an antitumor effect in BALB/C nude mice (Fig. 5A) [12]. To further confirm the role of CD8 + T cells, we blocked CD4 and CD8 with antibodies separately and collectively in the C57 mice tumor model to observe the antitumor effect of DC-TEX combined with PD-1 antibody under such circumstances. The antitumor effect was not affected after blocking CD4 (Fig. 5B), but disappeared after either blocking CD8 alone or both CD4 and CD8 simultaneously (Fig. 5C, D). This verified that the antitumor effect of DC-TEX combined with PD-1 antibody was mainly dependent on CD8 + T cells.

Combination of DC-TEX and PD-1 Ab suppresses tumor growth in a chemically induced HCC murine model

To determine if the antitumor effects of DC-TEX and PD-1 Ab combined therapy is independent of the creation methods of tumor models, we further evaluated whether the combined therapy could suppress tumor growth in a chemically induced HCC model. C57 mice born on the 15th day were intraperitoneally injected with DENA $50 \mathrm{mg} / \mathrm{kg}$ and then fed for 28 weeks prior to treatment. We divided the mice into six groups, including DC-TEX and PD-1 Ab groups ( $0 \mathrm{~h}, 72 \mathrm{~h}$ and $120 \mathrm{~h}$ groups), a DC-TEX group, a PD-1 Ab group, and a phosphate-buffered saline (PBS) group. The treatment schedule is shown in Supplementary Figure S10. The number of DC-TEX and the dosage of PD-1 Ab were as previously described. The DC-TEX group was injected intravenously and the PD-1 Ab group was injected intraperitoneally into a chemically induced HCC model three times, at 7-day intervals. At week 32, we performed immunohistochemical and 
flow cytometric analysis of the CD8 + T cells. We found that the $72 \mathrm{~h}$ group had significantly higher number of these cells than the other groups (Fig. 6A, B). Moreover, the level of AFP in the $72 \mathrm{~h}$ group was significantly lower than that in the other groups (Fig. 6C). The levels of IFN-y (Fig. 6D), IL-2, and TNF-a (Supplementary Figure S11) in the $72 \mathrm{~h}$ group were also significantly higher than those in the other groups. Liver weight was also significantly lower in the $72 \mathrm{~h}$ group than in the other groups (Fig. 6E,F).

\section{Discussion}

$\mathrm{HCC}$ is one of the most common primary liver tumors, with high recurrence and metastasis rates, and its incidence is increasing [20]. Currently, surgical resection remains the primary treatment option for patients with HCC. Unfortunately, the recurrence rate of HCC after surgical resection is still high. Besides, most patients are diagnosed at an advanced stage when treatment options are further limited and rarely effective.

Immunotherapy presents an apparent advantage for the treatment of HCC. For example, treatment with DC-based cancer vaccines has been demonstrated to be an appealing approach taking advantage of antitumor immune responses [21]. These responses primarily depend on cytotoxic T lymphocytes (CTLs) [22]. The number of tumor antigen-specific T cells is important for eliminating tumor cells, and in some studies this number aided in the prognosis of patients with HCC [23, 24]. In most cases, even though many tumor antigen-specific $T$ cells are present in tumor tissues, the tumors continue to grow. One of the primary explanations for this phenomenon is that the tumor microenvironment lessens the antitumor immune responses the tumor-antigen-specific T cells could have [25].

Some studies have demonstrated that tumor antigen-specific T cells suppress tumor growth in HCC [2628]. In these studies, antigen-specific $T$ cells were activated by tumor antigens and exhibited increased proliferation and cytokine secretion. These results demonstrated that the number of antigen-specific $T$ cells and the levels of cytokines were elevated significantly after receiving treatment. In our study, we observed that the number of CD8 $+T$ cells was significantly elevated after receiving DC-TEX treatment. We also found that the PD-1 + CD $8+T$ cells showed a similar increasing trend. This result is consistent with the fact that PD-1 can be inducted to express on T cells when T cells are stimulated by the antigen [29]. This also may explain the pressing question as to why significantly elevated $T$ antigen-specific $T$ cell population rarely promotes tumor regression $[30,31]$.

Some studies have confirmed that the PD-L1/PD-1 axis plays an important role in immune escape or negative immune regulation [32,33]. PD-1 presents mainly on activated T cells, binding to PD-L1 and inducing $T$ cell exhaustion. The antitumor effect of exhausted $T$ cells is significantly decreased. Blocking PD-1 and PD-L1 binding could restore the ability of exhausted CD8 + T cells to proliferate, secrete cytokines, and attack tumors with a remarkable and durable objective response [34, 35, 36]. In our study, the PD-1 antibody treatment group showed a distinct antitumor effect compared with PBS control group. This indicates that the PD-1 antibody could block PD-1 and PD-L1 binding in spontaneous antigen- 
specific CTLs. The number of CD8 + T cells was significantly increased compared with that in the PBS group, while the cytokine levels revealed similar increase.

PD-1 mRNA and protein expression are correlated with activation-induced apoptosis in murine thymocytes and shows time-dependent upregulation [37]. In our study, the timing of PD-1 antibody application after DC-TEX administration affected its efficacy. This indicated that the T cell exhaustion induced by DC-TEX could be restored by blocking the interaction between PD-1 and PD-L1. The cytokineand cytolytic activity-producing abilities of PD-1 + CD8 + T cells were weakened or inhibited when PD- $1+$ CD8 $+T$ cells were bound with PD-L1 in tumor tissue [38]. Fourcade et al. demonstrated that vaccines could activate tumor antigen-specific CD $8+T$ cells and induce more IFN-y production and higher lytic function. Meanwhile, PD-1 was upregulated in these antigen-specific CD $8+T$ cells. This indicates that upregulated expression of the inhibitory receptor PD-1 on antigen-specific CD $8+T$ cells was certainly correlated with treatment that was based on tumor vaccines [39].

Remarkably, a rational combination treatment consisting of DC-TEX activating tumor-specific T cells and PD-1 Ab inhibiting PD-1 checkpoint presented a superior antitumor response. Sierro et al. demonstrated that recombinant lentiviral vectors could induce specific antitumor CD $8+T$ cells, and that these specific antitumor CD $8+T$ cells also presented PD- 1 and failed to produce cytokines. Blocking the PD- 1 and PDL1 inhibitory pathway enhanced the efficacy of vaccination with recombinant lentiviral vectors in a mouse model [40]. After the PD-1 and PD-L1 inhibitory pathway was blocked by PD-1 antibody, the antitumor CD $8+T$ cells in a state of exhaustion could be rescued, and the cytokine production and proliferation were restored [41]. An important observation was that the tumor antigen-specific IFN- $y$ produced by $T$ cells plays an important role in determining the success of therapeutic tumor vaccines [42]. DC-TEX stimulates T cell proliferation and antitumor effects until T cell starts to exhaust.

Administration of PD-1 Ab before T cell exhaustion may not have any effect in aiding DC-TEX treatment. Therefore, we tested different timing of PD-1 Ab administration and found the timing is indeed critical for PD-1 Ab efficacy.

\section{Conclusion}

In conclusion, our study demonstrated that PD-1 antibody could elevate antitumor efficacy by restoring the exhausted tumor antigen-CD8+ T cells activated by DC-TEX. Interestingly, we found that the timing of PD-1 antibody administration has an important role in restoring the exhausted antigen-CD8+ T cells. The combination of DC-TEX and PD-1 antibodies showed superior results in inducing an antitumor response to HCC in mice.

\section{Abbreviations}

HCC, Hepatocellular carcinoma; TEXs, tumor cell-derived exosomes; DC-TEX, tumor cell-derived exosomes-pulsed dendritic cells; DCs, dendritic cells; PD-1, programmed death 1; PD-L1, programmed death ligand 1; DMEM,Dulbecco's modified Eagle's medium; FBS, fetal bovine serum; MRI, Magnetic 
Resonance Imaging; ELISAs, enzyme-linked immunosorbent assays; IL-2, interleukin-2; TNF-a, tumor necrosis factor-a; IFN- $\gamma$, interferon- $\gamma$; AFP, a-fetoprotein; PBS, phosphate-buffered saline; CTLs, cytotoxic T lymphocytes; CTLA-4, T-lymphocyte-associated protein 4.

\section{Declarations}

\section{Ethics approval and consent to participate}

Animal studies were approved by the Experimental Animal Management Committee of the Chinese Academy of Medical Sciences and Peking Union Medical College Institute of Biomedical Engineering.

\section{Consent for publication}

Not applicable.

\section{Availability of data and material}

The datasets supporting the conclusions of this article are included within the article and its additional files.

\section{Funding}

Financial Support: This work was supported by the Peking Union Medical College Innovation Research Team Fund, the Union Young Fund of Peking Union Medical College (3332016105) and the Sci-tech Development Project of Shandong Medicine and Health (2016WS0552) and the Natural Science Foundation of Shandong (ZR2017PH024) and the National Natural Science Foundation of China (81702821).

\section{Competing interests}

The authors declare that they have no competing interests.

\section{Authors' contributions}

Shengbin Shi and Cuijuan Wang performed the experiments, analyzed the data and prepared the manuscript. Chuangnian Zhang and Wenyu Zhang aided the data analysis and manuscript preparation. Yibo Qin contributed data analysis. Zuoxing Niu designed and supervised the study, analyzed the data, prepared and revised the manuscript. All authors read and approved the final manuscript.

\section{Acknowledgements}

Not applicable.

Conflict of interest: No author has any conflict of interest to declare. All authors have read the journal's policy on conflicts of interest and the journal's authorship agreement. 


\section{References}

1. Siegel R, Naishadham D, Jemal A. Cancer statistics, 2019. CA Cancer J Clin. 2019;69(1):7-34.

2. Jiajie Hou, Haiyan Zhang, Beicheng Sun, et al. The immunobiology of hepatocellular carcinoma in humans and mice: Basic concepts and therapeutic implications. J Hepatol.2020;72(1):167-182.

3. Timothy M Schmidt, L I Liu, Ivy E Abraham, et al. Efficacy and Safety of Sorafenib in a Racially Diverse Patient Population with Advanced Hepatocellular Carcinoma. Anticancer Res. 201।;38(7):4027-4034.

4. Sarun Juengpanich, Win Topatana, Chen Lu, et al. Role of cellular, molecular and tumor microenvironment in hepatocellular carcinoma: Possible targets and future directions in the regorafenib era. Int J Cancer. 2020;147(7):1778-1792.

5. Mercedes Iñarrairaegui, Ignacio Melero, Immunotherapy of Hepatocellular Carcinoma: Facts and Hopes. Clin Cancer Res.2018;24(7):1518-1524..

6. Zhen Lu, Bingfeng Zuo, Renwei Jing, et al. Dendritic cell-derived exosomes elicit tumor regression in autochthonous hepatocellular carcinoma mouse models. J Hepatol. 2017;67(4):739-748.

7. Qunfang Zhang, Haiding Huang, Fang Zheng, et al. Resveratrol exerts antitumor effects by downregulating CD8 + CD122 + Tregs in murine hepatocellular carcinoma. Oncoimmunology. 2020;9(1):1829346.

8. Haibo Zhou, Li Wu. The development and function of dendritic cell populations and their regulation by miRNAs. Protein Cell. 2017;8(7):501-513.

9. Merad M, Salmon H. A dendritic-cell brake on antitumour immunity. Nature. 2015;523:294-295.

10. Sangiliyandi Gurunathan, Min-Hee Kang, Muniyandi Jeyaraj, et al. Review of the Isolation, Characterization, Biological Function, and Multifarious Therapeutic Approaches of Exosomes. Cells. 2019;8(4):307.

11. Wolfers J, Lozier A, Raposo G, et al. Tumor-derived exosomes in cancer metastasis risk diagnosis and metastasis therapy. Nat Med. 2001;7:297-303.

12. Rao Q, Zuo B, Lu Z, et al. Tumor-Derived Exosomes Elicit Tumor Suppression in Murine Hepatocellular Carcinoma Models and Humans In Vitro. Hepatology. 2016;64(2):456-472.

13. Mohammad Davoodzadeh Gholami, Gholam Ali Kardar, Yousef Saeedi, et al. Exhaustion of T lymphocytes in the tumor microenvironment: Significance and effective mechanisms. Cell Immunol.2017;322:1-14.

14. Javier Celis-Gutierrez, Peter Blattmann, Yunhao Zhai, et al. Quantitative Interactomics in Primary T Cells Provides a Rationale for Concomitant PD-1 and BTLA Coinhibitor Blockade in Cancer Immunotherapy. Cell Rep. 2019;27(11):3315-3330.

15. Hyung-Don Kim, Gi-Won Song, Seongyeol Park, et al. Association Between Expression Level of PD1 by Tumor-Infiltrating CD8 + T Cells and Features of Hepatocellular Carcinoma. Gastroenterology.2018;155(6):1936-1950. 
16. David Kung-Chun Chiu, Vincent Wai-Hin Yuen, Jacinth Wing-Sum Cheu, et al. Hepatocellular Carcinoma Cells Up-regulate PVRL1, Stabilizing PVR and Inhibiting the Cytotoxic T-Cell Response via TIGIT to Mediate Tumor Resistance to PD1 Inhibitors in Mice. Gastroenterology.2020;159(2):609-623.

17. Julien Fourcade, Zhaojun Sun, Mourad Benallaoua, et al. Upregulation of Tim-3 and PD-1 expression is associated with tumor antigen-specific CD8+ T cell dysfunction in melanoma patients. J Exp Med.2010;207(10):2175-86.

18. Xiaodong Wang, Xiaohui Yang, Chang Zhang, et al. Tumor cell-intrinsic PD-1 receptor is a tumor suppressor and mediates resistance to PD-1 blockade therapy. Proc Natl Acad Sci U S A. 2020;117(12):6640-6650.

19. Tumeh PC, Harview CL, Yearley JH, et al. PD-1 blockade induces responses by inhibiting adaptive immune resistance. Nature. 2014;515:568-571.

20. Lindsey A Torre, Freddie Bray, Rebecca L Siegel, et al. Global cancer statistics, 2012. CA Cancer J Clin. 2015;65(2):87-108.

21. Stefanie K Wculek, Francisco J Cueto, Adriana M Mujal, et al. Dendritic cells in cancer immunology and immunotherapy. Curr Opin Immunol. 2014;27:26-32.

22. Copier J, Dalgleish A. Overview of tumor cell-based vaccines. Nat Rev Immunol.2020;20(1):7-24.

23. Li G, Liu D, Cooper TK, et al. Successful chemoimmunotherapy against hepatocellular cancer in a novel murine model. $J$ Hepatol. 2017;66:75-85.

24. Brunner SM, Rubner C, Kesselring R, et al. Tumor-infiltrating, interleukin-33-producing effectormemory CD8(+) T cells in resected hepatocellular carcinoma prolong patient survival. Hepatology. 2015;61:1957-1967.

25. S Di Blasio, G F van Wigcheren, A Becker, et al. The tumour microenvironment shapes dendritic cell plasticity in a human organotypic melanoma culture. Nat Commun. 2020;11(1):2749.

26. Chen Lu, Dawei Rong, Betty Zhang, et al. Current perspectives on the immunosuppressive tumor microenvironment in hepatocellular carcinoma: challenges and opportunities. $\mathrm{Mol}$ Cancer.2019;18(1):130.

27. Peter G Hendrickson, Michael Olson, Tim Luetkens, et al. The promise of adoptive cellular immunotherapies in hepatocellular carcinoma. Oncoimmunology. 2019;9(1):1673129.

28. Flecken T, Schmidt N, Hild S, et al. Immunodominance and Functional Alterations of TumorAssociated Antigen-Specific CD8+ T-Cell Responses in Hepatocellular Carcinoma. Hepatology. 2014;59:1415-1426.

29. Agata, YA, Kawasaki, H. Nishimura, et al. Expression of the PD-1 antigen on the surface of stimulated mouse T and B lymphocytes. Int Immunol. 1996 May;8(5):765-72. 1996.

30. Rosenberg SA, Yang JC, Restifo NP. Cancer immunotherapy: moving beyond current vaccines. Nat Med. 2004;10:909-915.

31. Nicolas Jacquelot, Takahiro Yamazaki, Maria P Roberti, et al. Sustained Type I interferon signaling as a mechanism of resistance to PD-1 blockade. Cell Res. 2019 Oct;29(10):846-861. 
32. Hailemichael $Y$, Dai Z, Jaffarzad N, et al. Persistent antigen at vaccination sites induces tumorspecific CD8(+) T cell sequestration, dysfunction and deletion. Nat Med. 2013;19:465-472.

33. Danilova L, Wang H, Sunshine J, et al. Association of PD-1/PD-L axis expression with cytolytic activity, mutational load, and prognosis in melanoma and other solid tumors. Proc Natl Acad Sci US A. 2016;113:E7769-E7777.

34. Mariathasan S, Turley SJ, Nickles D,et al. TGF $\beta$ attenuates tumour response to PD-L1 blockade by contributing to exclusion of T cells. Nature. 2018;554(7693):544-548.

35. Li G, Liu D, Cooper TK, et al. Successful chemoimmunotherapy against hepatocellular cancer in a novel murine model. J Hepatol. 2017;66:75-85.

36. Sprinzl MF, Galle PR. Current progress in immunotherapy of hepatocellular carcinoma. J Hepatol. 2017;66:482-484.

37. R Vibhakar, G Juan, F Traganos, et al. Activation-Induced Expression of Human Programmed Death-1 Gene in T-Lymphocytes. Exp Cell Res.1997; 232 (1):25-8.

38. Wu X, Zhang H, Xing Q, et al. PD-1(+) CD8(+) T cells are exhausted in tumours and functional in draining lymph nodes of colorectal cancer patients. Br J Cancer. 2014;111:1391-1399.

39. Fourcade J, Sun Z, Pagliano O, et al. PD-1 and Tim-3 regulate the expansion of tumor antigenspecific CD8+ T cells induced by melanoma vaccines. Cancer Res, 2014;74:1045-1055.

40. Sierro SR, Donda A, Perret R, et al. Combination of lentivector immunization and low-dose chemotherapy or PD-1/PD-L1 blocking primes self-reactive T cells and induces antitumor immunity. Eur J Immunol. 2011;41:2217-2228.

41. Yuki Inada, Eishiro Mizukoshi, Takuya Seike,et al. Characteristics of Immune Response to TumorAssociated Antigens and Immune Cell Profile in Patients With Hepatocellular Carcinoma. Hepatology. 2019;69(2):653-665.

42. Finck $\mathrm{A}$, Gill $\mathrm{SI}$, June $\mathrm{CH}$. Cancer immunotherapy comes of age and looks for maturity. Nat Commun. 2020;11(1):3325.

\section{Figures}



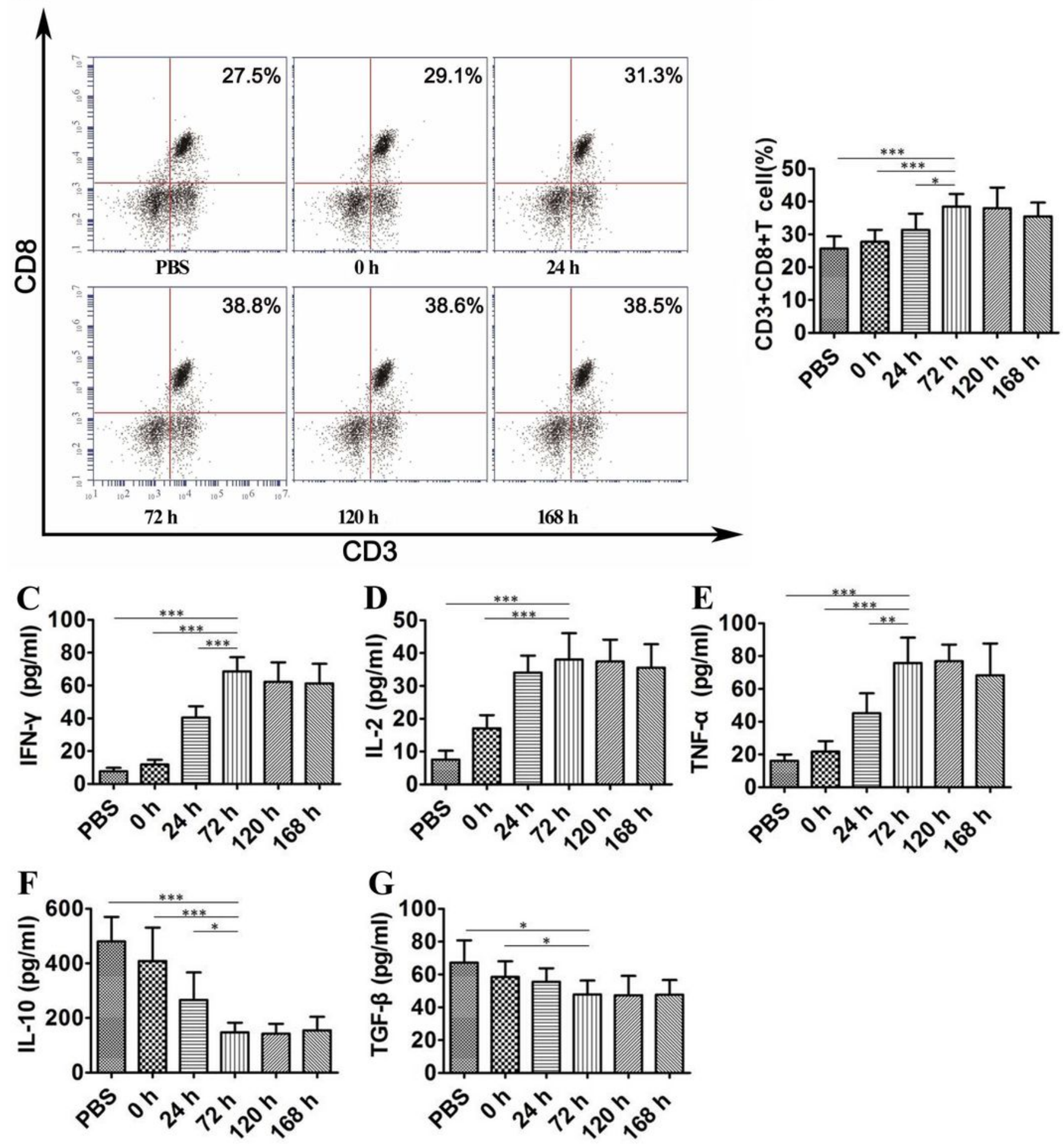

Figure 1

CD8+ T cell numbers and cytokine production levels increased after DC-TEX injection in vivo. A. The number of CD8+ T cells in tumor tissues. After injecting DC-TEX, we collected CD8+ T cells at $0,24,72$, 120 , and $168 \mathrm{~h}$ by flow cytometry. B-D. Serum IFN- $y$, IL-2 and TNF-a levels were analyzed by ELISA. The process of T-cell activation produced several cytokines. IFN- $y$, IL-2 and TNF-a levels were significantly different at 0, 24 and $72 \mathrm{~h}$ but were not significantly increased at 120 and $168 \mathrm{~h}$. E, F. Serum IL-10 and 
TGF- $\beta$ were significantly different at 0,24 and $72 \mathrm{~h}$ but were not significantly decreased at 120 and $168 \mathrm{~h}$ ( $n=6$ mice/group, $\left.{ }^{* \star *} P<0.001\right)$. ( $n=6$ mice/group, $\left.{ }^{*}<<0.05 * \star, P<0.01,{ }^{* \star *} P<0.001\right)$.

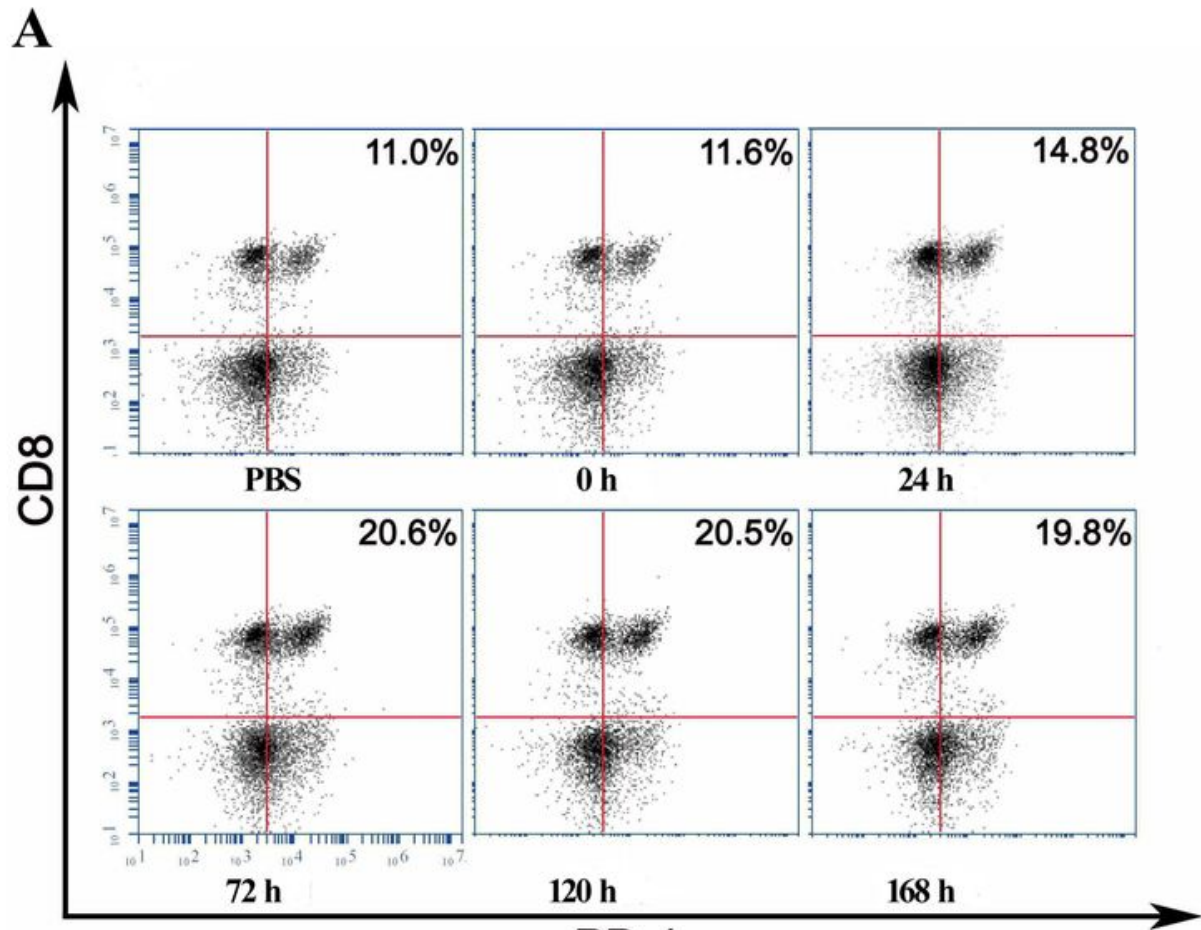

PD-1

$\mathbf{C}$

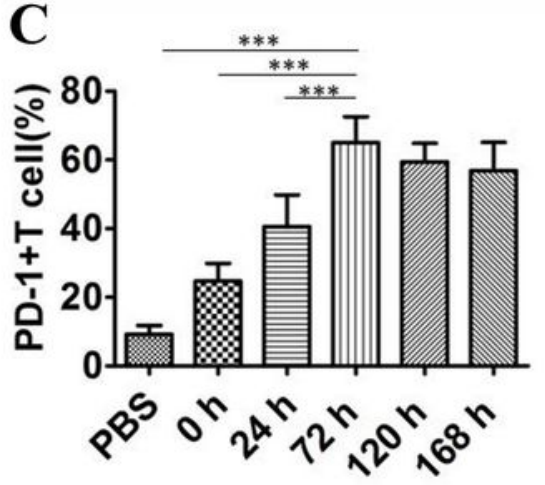

B

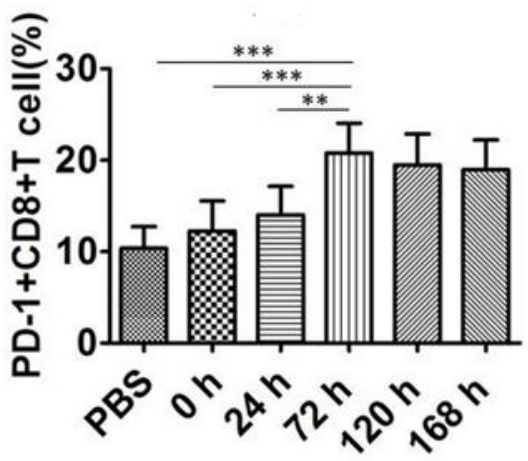

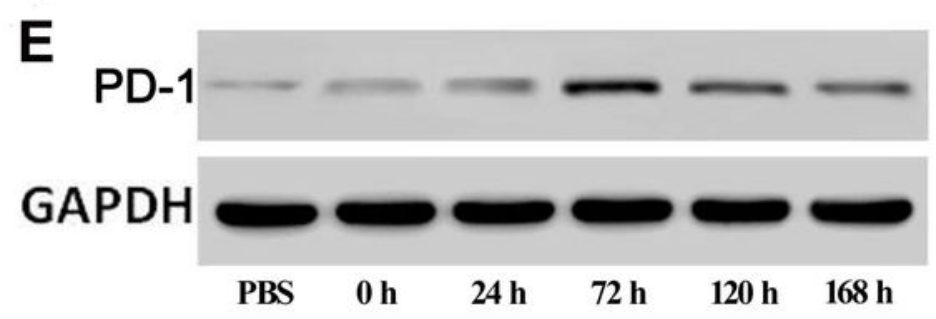

Figure 2

The variation of PD-1 expression in CD8+ T cells. A, B. The number of PD-1+CD8+ T cells in tumor tissues at different time points. The trend showed that the number of PD-1+CD8+ T cells increased significantly from 0 to $72 \mathrm{~h}$ but did not further increase from $72 \mathrm{~h}$ to $168 \mathrm{~h}(\mathrm{P}>0.05)$. ( $\mathrm{n}=6 \mathrm{mice} / \mathrm{group}$, 
$\left.{ }^{\star} * P<0.01,{ }^{* \star *} P<0.001\right)$. C. T cells derived from the spleen of C57 mice were co-cultured with DC-TEX, and the number of PD- $1+T$ cells showed a significant difference from 0 to $72 \mathrm{~h}(\star \star \star \mathrm{P}<0.001)$. D. The mRNA of PD-1 showed higher expression at $72 \mathrm{~h}$ by $\mathrm{qPCR}\left({ }^{\star} P<0.05,{ }^{\star *} \mathrm{P}<0.01,{ }^{\star \star *} \mathrm{P}<0.001\right)$. E T cells and DC-TEX were co-cultured and showed the higher expression of PD-1 at $72 \mathrm{~h}$ by western blotting.

A

PBS

DC-TEX

PD-1 Ab

o h

$24 \mathrm{~h}$

$72 \mathrm{~h}$

$120 \mathrm{~h}$

$168 \mathrm{~h}$
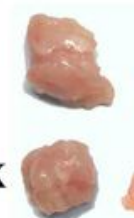

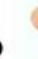
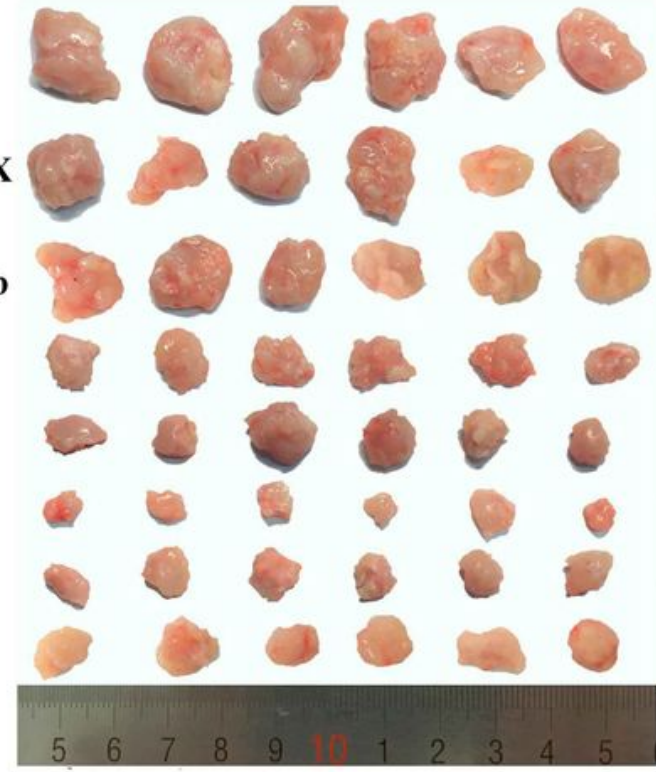

C

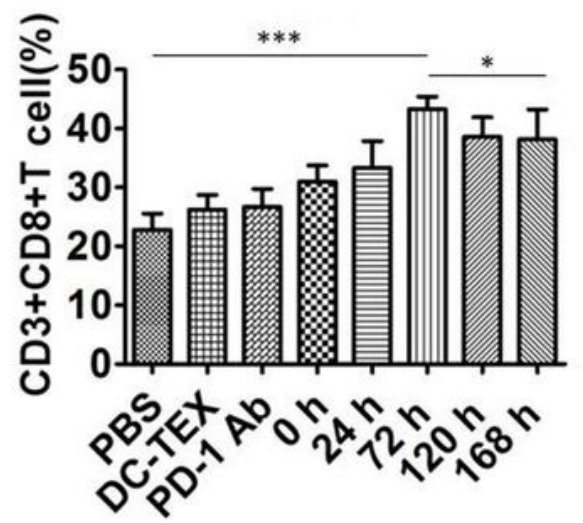

D

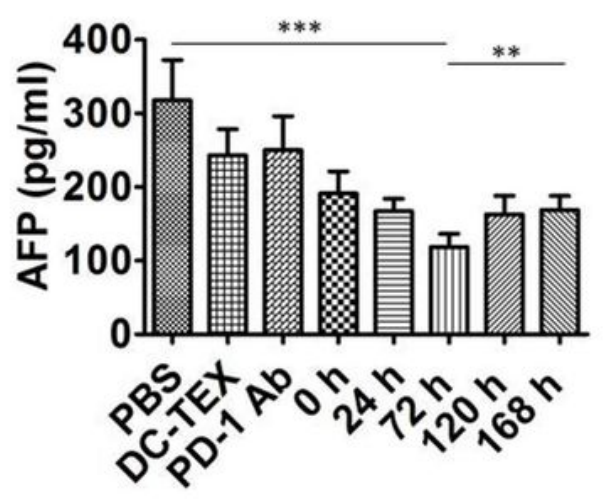

E

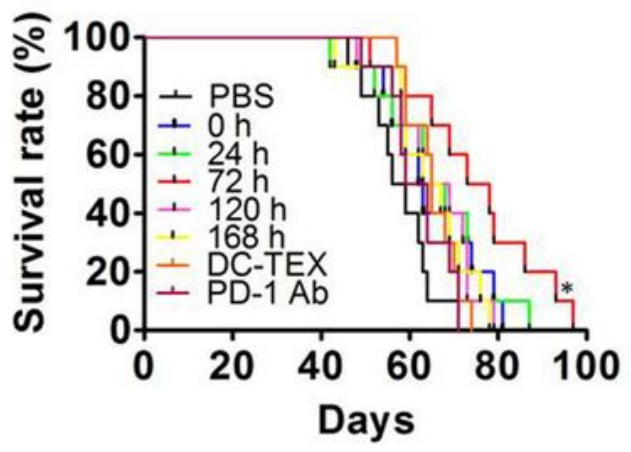

Figure 3 
The treatment was PD-1 Ab and DC-TEX applied at different times resulted in different treatment efficacy. A-B. Tumor weight were measured in each group on day 40 after implantation. Tumor weights were significantly lower in the $72 \mathrm{~h}$ group than in the other groups. C. The number of CD8+ T cells in tumor tissues was detected by flow cytometry. D. The AFP level in serum was analyzed using an ELISA kit. The AFP level was was significantly lower in the $72 \mathrm{~h}$ group than the other groups. E. The survival times of all groups were analyzed using the Kaplan-Meier method. ( $n=6$ mice/group, ${ }^{*} P<0.05$, ${ }^{* *} P<0.01,{ }^{* \star *} P<0.001$ ).

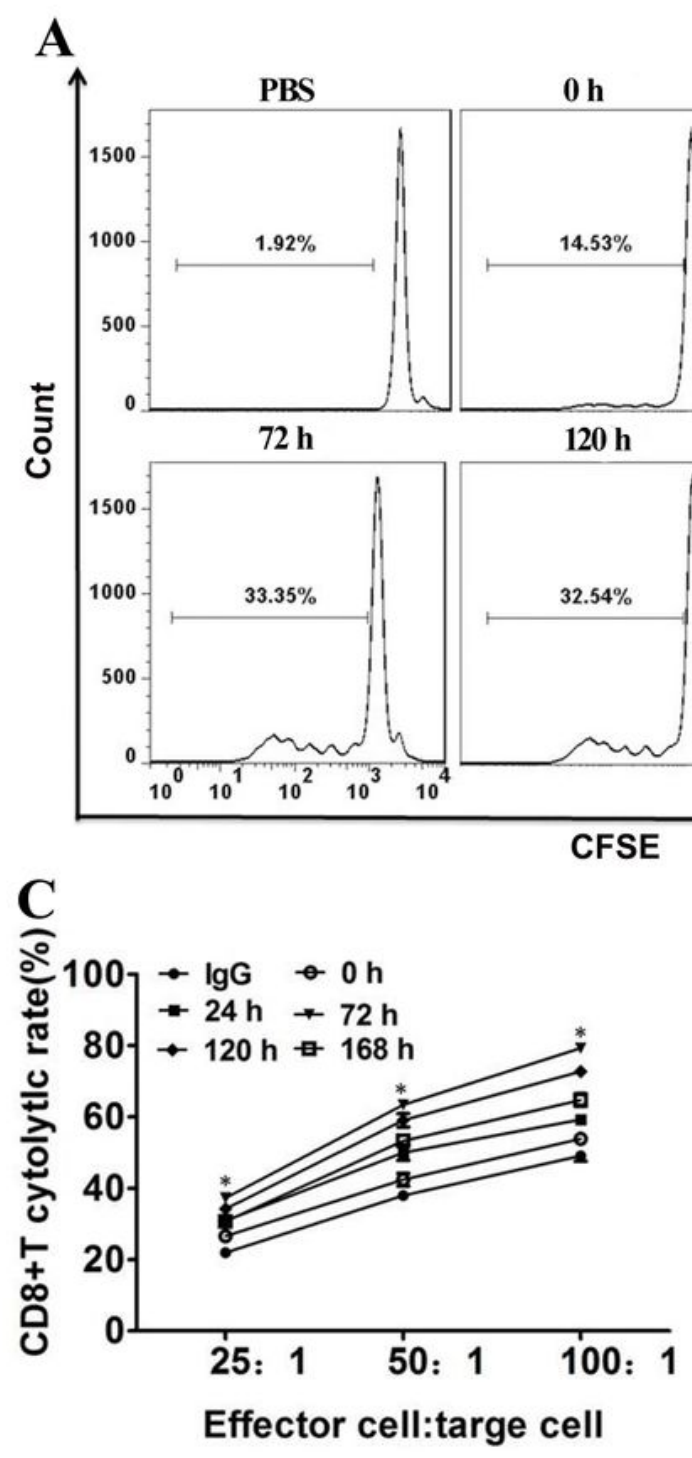

B

D
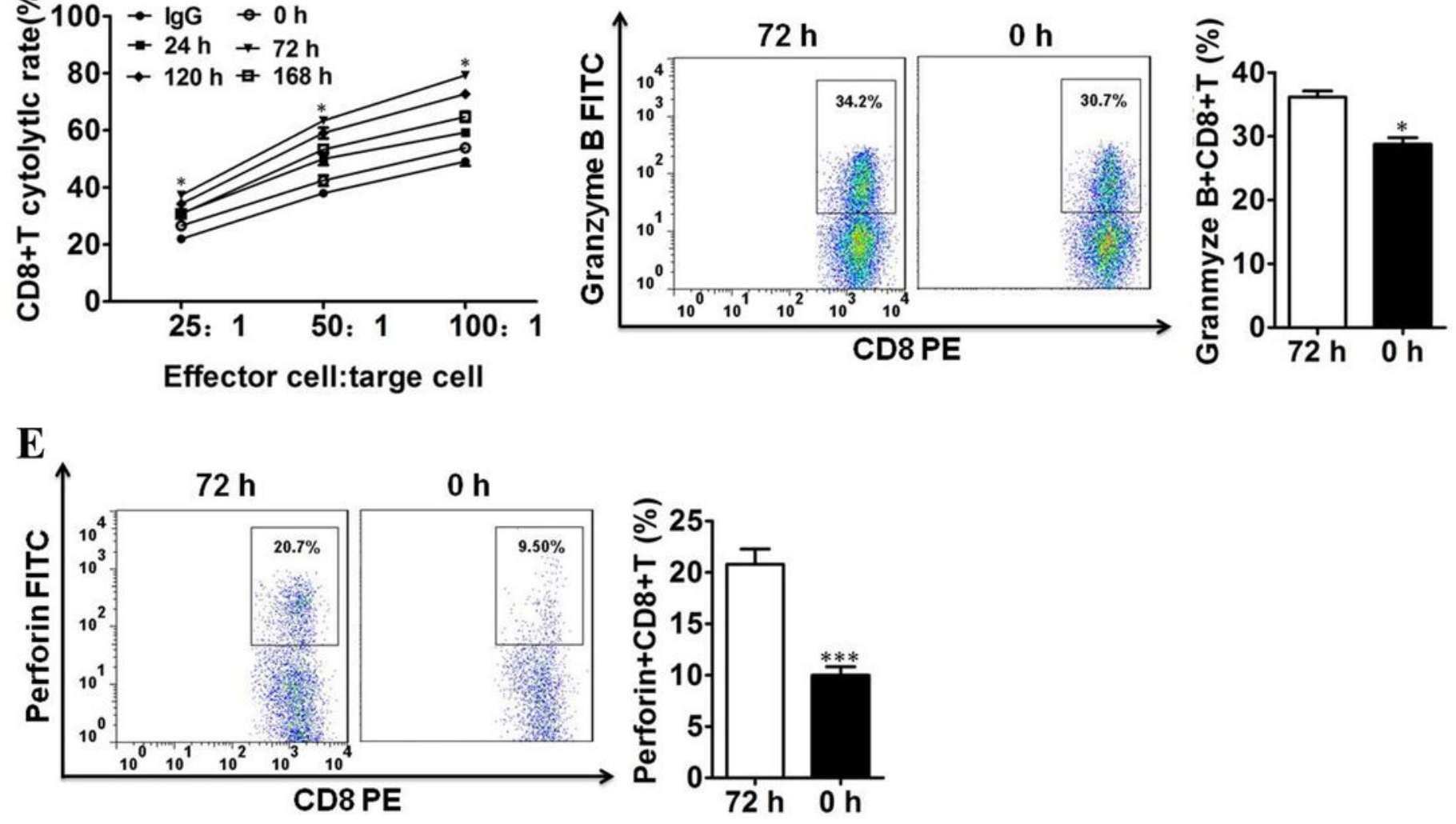

Figure 4 
The proliferative ability of PD-1+CD8+ T cells was affected by PD-1 Ab in vitro. A-B.The PD-1+CD8+ T cells were labeled with CFSE and then cocultured with PD-1 Ab $(20 \mu \mathrm{g} / \mathrm{ml})$ to analyze their proliferative ability by flow cytometry. C. Cytolysis assay for CD 8+ T cells activated by DC-TEX plus PD-1 Ab at different point against Hepa1-6 cells at different ratios. D, E. Analysis of granzyme $B+C D 8+T$ cells and perforin $+C D 8+T$ cells by flow cytometry. $\left({ }^{\star} P<0.05\right.$, $\left.{ }^{\star \star *} P<0.001\right)$.
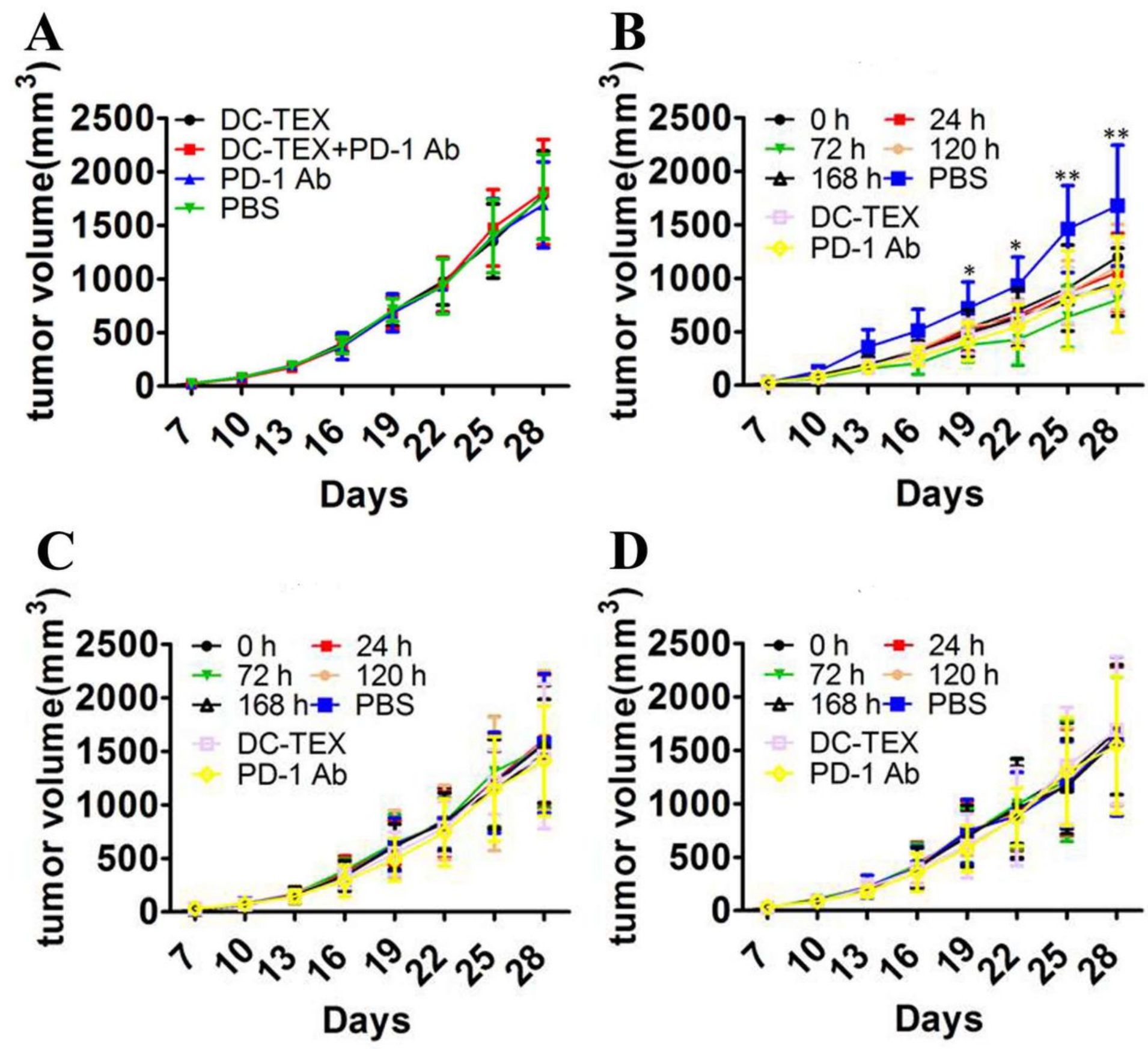

Days

Figure 5

The antitumor effect of DC-TEX and PD-1 Ab was mediated by CD8+ T cells. Figure A to D shows tumor volume measurements post treatment. Figure $A$ used $\mathrm{HCC}$ model in $\mathrm{BALB} / \mathrm{c}$ nude mice and $\mathrm{B}$ to $\mathrm{D}$ used HCC model established in C57 mice. A. DC-TEX and PD-1 Ab do not have antitumor effect in T cell- 
deficient BALB/c nude mice. B.CD 4 antibody was used on days $-2,-1$, and 0 to block CD4+T cells before DC-TEX and PD-1 Ab treatment. C. CD8 antibody was used on days $-2,-1$, and 0 to block CD8+T cells before DC-TEX and PD-1 Ab treatment. D. CD4 and CD 8 antibodies were simultaneously used on days -2 , -1 , and 0 to block CD4+T cells and CD8+T cells before DC-TEX and PD- 1 Ab treatment. $(n=6$ mice/group, ${ }^{*} \mathrm{P}<0.05$, $\left.{ }^{* *} \mathrm{P}<0.01,{ }^{* * *} \mathrm{P}<0.001\right)$.

A
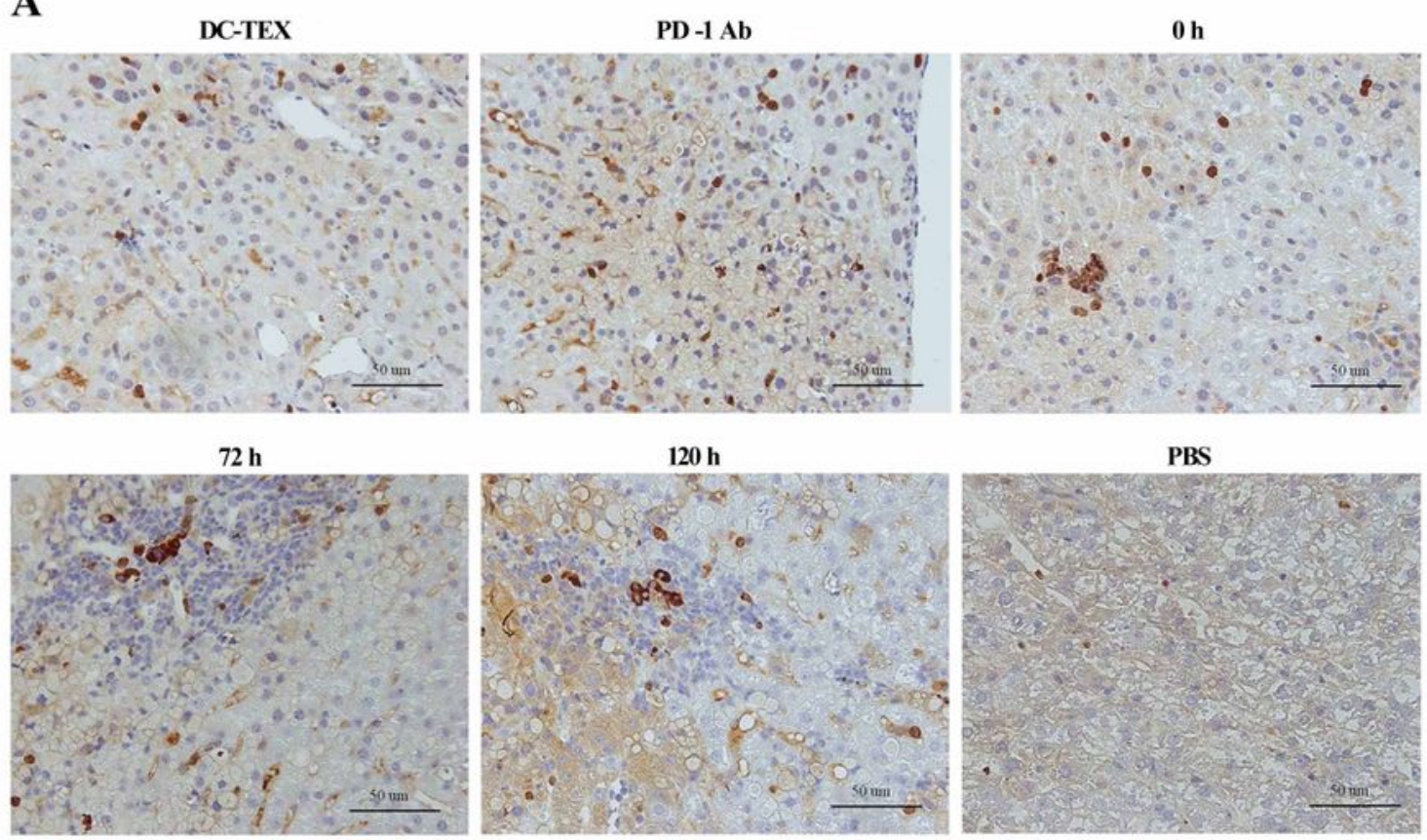

B

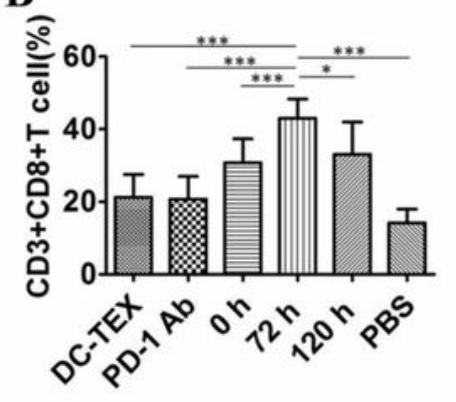

C

D
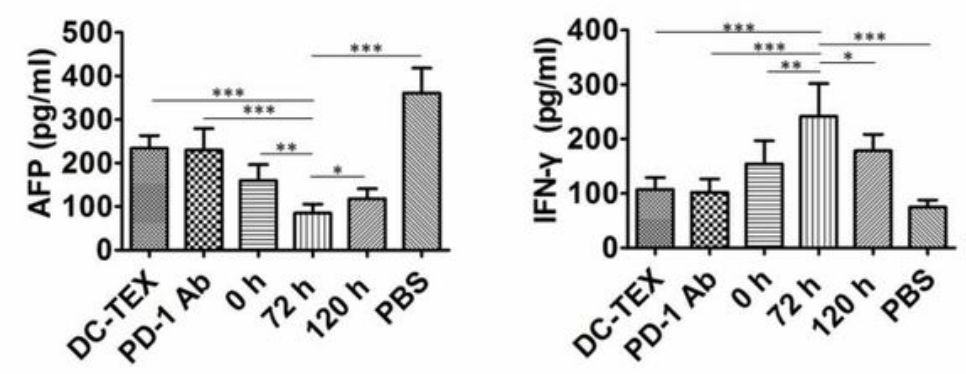

$\mathbf{E}$

$\mathbf{F}$
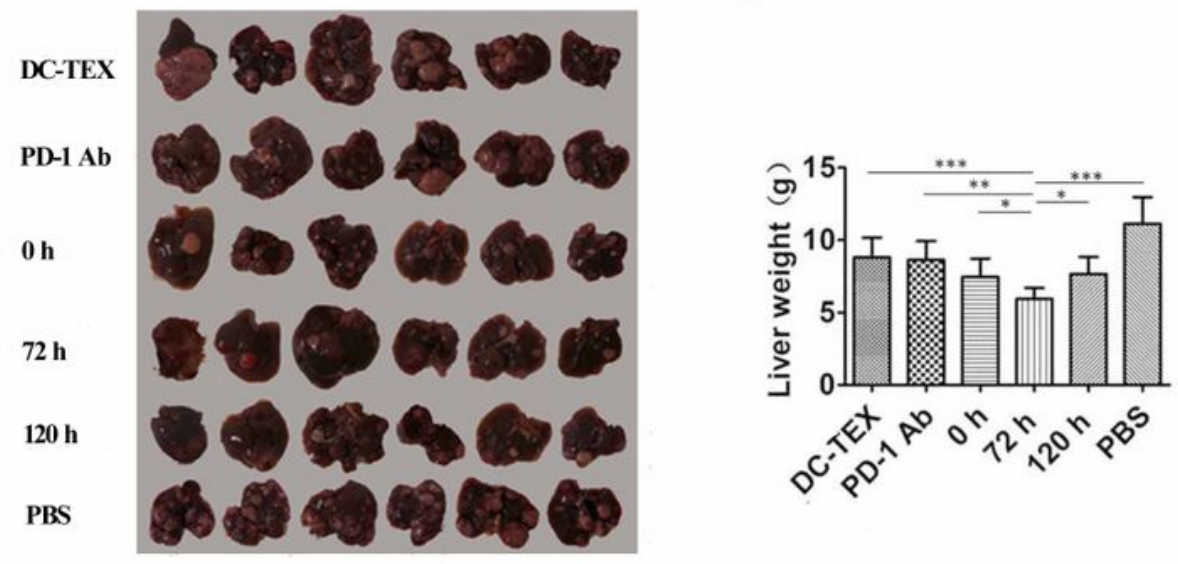

Figure 6 
The antitumor effect of DC-TEX and PD-1 Ab in a chemically induced HCC model. A. The immunohistochemistry of CD8+T cells in tumor tissues (scale bar, $50 \mu \mathrm{m}$ ). B. CD8+T cells in tumor tissues were analyzed by flow cytometry. The number of CD8+T cells in the $72 \mathrm{~h}$ group was significantly increased. C. Cytokine IFN-y levels were significantly higher in the $72 \mathrm{~h}$ group than in other groups, as assessed by ELISA. D. AFP levels differed among groups. AFP levels, as assessed by ELISA, were significantly lower in the $72 \mathrm{~h}$ group than in other groups. E. Measurements of liver weights in mice. $(\mathrm{n}=6$ mice/group, $\left.{ }^{*} P<0.05 * \star, P<0.01, * \star * P<0.001\right)$.

\section{Supplementary Files}

This is a list of supplementary files associated with this preprint. Click to download.

- SupplementaryInformation.docx 